\title{
Estimación sectorial de la huella hídrica de la ciudad de Bogotá generada en el año 2014
}

\section{Sectorial estimate of the water footprint of Bogotá city generated in the year 2014}

\author{
Ángela Castillo-Rodríguez ${ }^{1}$, Marisol Castro-Chaparro ${ }^{2}$, Álvaro Gutiérrez-Malaxechebarría ${ }^{3}$, Carmen Aldana- \\ Gaviria $^{4}$

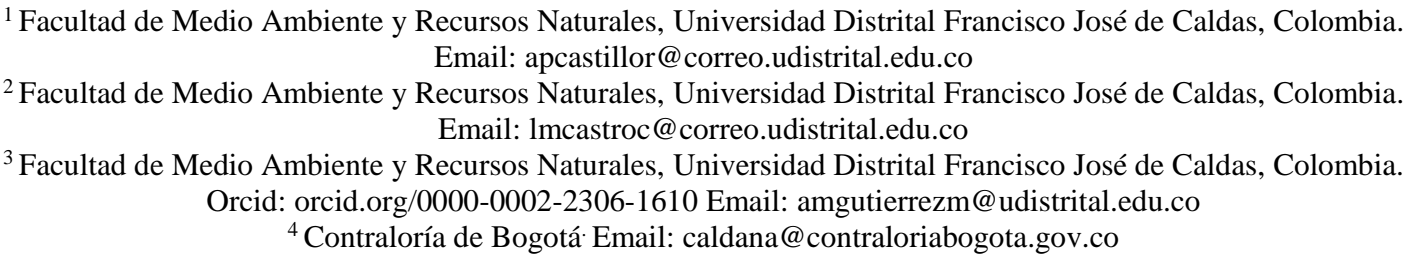

Recibido: octubre 19, 2017. Aceptado: diciembre 04, 2017. Versión final: febrero 20, 2018.

\begin{abstract}
Resumen
El presente artículo tiene como propósito presentar la estimación de la huella hídrica de la ciudad de Bogotá generada en el año 2014. La huella se define, de manera simplificada, como un indicador de consumo y contaminación de agua dulce. Para ello fueron seleccionados cuatro sectores para evaluar el tipo de huella correspondiente en cada uno: doméstico, industrial, agrícola y residuos sólidos. Se siguió la metodología propuesta por Hoekstra et al. La huella hídrica total de los sectores evaluados se estimó en 9.489,58 millones $\mathrm{de}^{3}$, donde el sector de mayor huella fue el agrícola con 4.555,14 millones de $\mathrm{m}^{3}$, seguido por los sectores doméstico, de residuos e industrial. Finalmente, es posible concluir que la huella hídrica es insostenible, y que, por lo tanto, la ciudad es vulnerable frente a la disponibilidad del recurso.
\end{abstract}

Palabras clave: Bogotá; huella hídrica; sostenibilidad; consumo de agua; gestión del agua.

\begin{abstract}
This article presents the estimate of the Water Footprint of Bogota city generated in the year 2014. The water footprint is defined, in simple terms, as an indicator of consumption and pollution of freshwater. Four sectors were selected to evaluate the type of corresponding footprint in each of them: domestic, industrial, agricultural and solid waste. The methodology followed was proposed by Hoekstra et al. The total water footprint of the evaluated sectors was estimated at $9.489,58$ million $\mathrm{m}^{3}$, where the sector of higher footprint was the agricultural one with $4.555,14$ million $\mathrm{m}^{3}$, followed by the solid waste and industrial sectors. Finally, it can be concluded that the footprint is unsustainable and, therefore, the city is vulnerable considering the availability of the hydric resource.
\end{abstract}

Keywords: Bogotá; water footprint; sustainability; water consumption; production; water management.

ISSN impreso: 1657 - 4583, ISSN en línea: 2145 - 8456

Este artículo puede compartirse bajo la licencia CC BY-ND 4.0 y se referencia usando el siguiente formato: A. Castillo-Rodríguez, M. CastroChaparro, A. Gutiérrez-Malaxechebarría, C. Aldana-Gaviria, "Estimación sectorial de la huella hídrica de la ciudad de Bogotá generada en el año 2014," Rev. UIS Ing., vol. 17, no. 2, pp. 19-32, 2018. Doi: https://doi.org/10.18273/revuin.v17n2-2018002 


\section{Introducción}

El agua está involucrada directamente en todas las actividades realizadas en una ciudad, y, por tanto, la huella hídrica es una herramienta útil para la formulación de medidas de mitigación y adaptación que permitan mejorar los niveles de sostenibilidad de las actividades humanas, ya que el objetivo de este indicador es medir la intensidad de uso del recurso [1]. En síntesis, la huella hídrica es el volumen total de agua utilizado directa o indirectamente para producir cualquier bien o servicio. Según Arévalo [2], este indicador hace posible apreciar la presión ejercida por las actividades domésticas, industriales y agrícolas, entre otras, además facilita la evaluación de los diferentes modelos de consumo y sus posibilidades de evolución en función de ese recurso natural.

La huella hídrica tiene tres componentes: el agua azul, definida como el agua proveniente de fuentes superficiales o subterráneas; el agua lluvia almacenada por la vegetación, y, finalmente, el agua gris, que se refiere a los volúmenes de agua necesarios para asimilar la carga contaminante, con base en las normas de calidad ambiental [3], se recomienda estimarla bajo diferentes parámetros de calidad donde la máxima de estas estimaciones sea la huella gris definitiva [4]. En este caso no se analiza la vulnerabilidad asociada a la contaminación subterránea, ni se hacen modelaciones, contrario a lo presentado por Gómez-Isidro, GutiérrezLozano y Torres [5] y por Blanco, Donado y Barajas[6]. Además, se incluye el concepto de agua virtual, que hace referencia al agua proveniente de territorios externos al área evaluada. Este concepto, permite incluir volúmenes de agua que asocian directamente actividades productivas foráneas, en los términos económicos internos del área geográfica que se desea estudiar, y, en consecuencia, permite un balance comercial referente al recurso hídrico [7].

Como menciona la WWF [8] la metodología empleada para el cálculo de la huella hídrica fue propuesta en 2002 por Arjen Y. Hoekstra del grupo UNESCO - IHE. Posteriormente fue desarrollada por la Universidad de Twente, y, en la actualidad, su descripción detallada aparece en varios manuales de la Water Footprint Network[8]. Para la aplicación de este indicador en la ciudad de Bogotá se empleó una adaptación de esta metodología que facilita la formulación de políticas, programas y proyectos encaminados a mejorar la gestión del agua.

La presente evaluación incluye cuatro sectores, el doméstico, que fue analizado mediante un enfoque de consumo; el industrial que emplea alrededor del $20 \%$ del agua total consumida en un país, que equivale a un consumo de $130 \mathrm{~m}^{3}$ /persona /año, según Conagua [9]. Así mismo, se tuvo en cuenta el sector agrícola mediante el consumo de productos, lo que implica aportes de agua virtual que se encuentra constituida por los tres componentes mencionados anteriormente[4]. En el caso de animales vivos, el agua azul corresponde al agua de consumo, a la utilizada para limpiar el corral y lavar el animal, entre otras cosas; el agua verde está relacionada con el consumo de alimentos de los animales, y el agua gris se asocia con la lixiviación, y el escurrimiento de nitrógeno en actividades pecuarias. Mientras que para los productos agrícolas el agua azul corresponde a las actividades de riego; el agua verde, al agua lluvia incorporada por los cultivos, y el agua gris a la lixiviación de agroquímicos y fertilizantes [4]. Por último, se tuvo en cuenta la huella hídrica asociada a la producción de lixiviados en el relleno sanitario Doña Juana como consecuencia de la descomposición de los residuos sólidos.

Una vez cuantificada la huella hídrica total mediante los resultados de los diferentes sectores, se realizó la evaluación su sostenibilidad. Esta evaluación arroja información importante respecto al impacto real sobre el recurso, debido a que compara la disponibilidad de este con los niveles de consumo y contaminación generados en el periodo de evaluación.

Teniendo en cuenta lo anterior, junto con los resultados desagregados de los sectores, es posible emplear la huella hídrica como herramienta para comparar los niveles de uso y consumo mediante la huella per cápita con países como Canadá o China, que cuentan con huellas por habitante de 2. 048,87 y 702,48 $\mathrm{m}^{3} /$ año respectivamente, según Conagua [10]. Además, es una herramienta para el desarrollo de propuestas tendientes a mejorar la sostenibilidad de las actividades antrópicas, con base en la interacción existente entre las relaciones sociales, los recursos económicos y los recursos naturales. Esto permite comprender al ambiente como un sistema complejo que requiere monitoreo permanente de su estado, a fin de desarrollar y realizar actividades que procuren su correcta utilización y su conservación.

\section{Materiales y métodos}

La metodología empleada para estimación de la huella hídrica $(\mathrm{HH})$ de Bogotá se basa en la propuesta de Hoekstra [4], ya que facilita la evaluación del uso y del consumo de agua en ciudades. Para la cuantificación de la huella en áreas geográficamente definidas, esta metodología presenta la siguiente ecuación, en la que se incluyen los diferentes procesos o sectores presentes en la ciudad: 


$$
\boldsymbol{W F}_{\text {área }}=\sum W F \operatorname{Froc}[q]
$$

Donde:

WF]_área: huella hídrica del área geográficamente definida.

WFproc: huella hídrica de los diferentes procesos o sectores.

La cuantificación del consumo de agua en los diferentes procesos está dada por las siguientes ecuaciones y planteamientos.

\subsection{Sector doméstico}

La estimación de la huella hídrica para el sector doméstico se realizó desde el enfoque del consumo, ya que no se encuentran actividades productivas o de proceso asociadas al consumo. Para este sector se realizó el cálculo de las huellas hídricas azul y gris, para, finalmente, realizar su sumatoria y así obtener la estimación total del sector.

\subsubsection{Huella hídrica azul del sector doméstico}

Según Hoekstra [4] se refiere al consumo de los recursos de agua azul, es decir, de agua superficial y subterránea. "El consumo" se refiere a la pérdida de agua de la masa de agua disponible en un área de captación. La huella hídrica azul del sector doméstico se estima para un tiempo determinado, como lo plantea la Water Footprint Network [4]:

$$
\text { 【WFD】_Azul }=\text { afluente }- \text { efluente }
$$

Donde:

WFDAzul: huella hídrica azul del sector doméstico

Afluente: es el volumen de agua usada en la actividad evaluada, como aparece en la factura de servicios de agua potable.

Efluente: es el volumen de agua calculada.

Con base en la delimitación geográfica de la ciudad y según la idea de que "el agua que no vuelve a la misma zona de flujo, por ejemplo, es devuelta a otra zona de captación o al mar..." [4], (para la ciudad de Bogotá las cuencas abastecedoras y de recepción de vertimientos no son las mismas), se puede asumir que el efluente de la cuenca es cero. El desarrollo de la ecuación es de la siguiente manera:

$$
\begin{aligned}
& \text { [WFD)_Azul=afluente }=[\mathrm{VF}) \\
& \text { _EAB+VNF+V_AB+[GP }] \text { _PTAP+V_SC }
\end{aligned}
$$

Donde:

VFEAB = volumen de agua facturado por la empresa de acueducto y alcantarillado de Bogotá (EAB).

VNF = volumen de agua no facturado. calculado como la diferencia entre el volumen de agua suministrado a bogotá y el volumen facturado por la EAB. Estas perdidas se generan en los túneles y las tuberías que distribuyen el agua a los diferentes usuarios de la ciudad, ya sea por fugas en el sistema o por acciones ilegales.

$\mathrm{VAB}=$ volumen vendido de agua en bloque.

GPPTAP = gasto de potabilización en las plantas de tratamiento de agua potable (PTAP). Se refiere al volumen de agua utilizado en plantas de tratamiento para el mantenimiento y el funcionamiento de la planta, principalmente del lavado de filtros y de los drenajes de los sedimentadores y los floculadores.

VSC = volumen de agua subterránea concesionada por la secretaría distrital de ambiente (SDA) y la Corporación Autónoma Regional de Cundinamarca - CAR

\subsubsection{Huella hídrica gris del sector doméstico}

La huella hidrica gris se refiere a la contaminación y está definida como el volumen de agua dulce que se requiere para asimilar una carga de contaminantes, segun las concentraciones naturales y estándares ambientales de calidad de agua. Es recomendable que la cuantificación se realice bajo diferentes parámetros de calidad. La huella hídrica gris será la máxima entre las huellas hídricas grises calculadas, como los presenta WFN.

En este caso se evaluó la huella hídrica gris para los parámetros de demanda biológica de oxigeno (DBO5) y sólidos suspendidos totales (SST) de los vertimientos generados por el sector, de los cuales el resultado de mayor magnitud corresponderá a la huella hídrica gris del sector doméstico.

Según Hoekstra [4] la huella hidrica gris se estima de la siguiente manera:

$$
\begin{aligned}
& \text { [WFD } \rrbracket \_(\text {Gris })=\mathrm{L} /\left(\mathrm{C} \_ \text {max }-\mathrm{C} \_ \text {nat }\right)=\left(\text { ( }(\mathrm{Vol}) \rrbracket \_\right. \text {efl x } \\
& \text { C_efl)-( [Vol】_afl x C_afl))/(C_max-C_nat })
\end{aligned}
$$


22 RUIIS REVISTA UIS

Donde:

WFDGris: huella hídrica gris del sector doméstico.

Volefl: corresponde al volumen estimado de vertimientos domésticos que son dispuestos en el alcantarillado. Este volumen corresponde aproximadamente al $85 \%$ del agua de uso doméstico[11]. De este porcentaje el $30 \%$ es transportado a la cuenca del río Salitre para su posterior tratamiento y el $70 \%$ restante es vertido sin tratamiento a las cuencas de los ríos Tunjuelo y Fucha, afluentes del río Bogotá [12], lo cual diferenciará el volumen del efluente y su respectiva carga para calcular la huella hidríca gris del sector, así:

WFD】_(Gris $)=(($ (Vol】_eflST x C_eflST $)+\llbracket(\mathrm{Vol}$

】_eflPTAR $\quad \mathrm{x} \quad \mathrm{C} \_$eflPTAR $\left.)\right)-\left(\llbracket \mathrm{Vol} \rrbracket \_a f l \quad \mathrm{x}\right.$

C_afl))/(C_max-C_nat )

Donde:

VolefIST: volumen de agua vertido sin tratamiento a las cuencas de los ríos Tunjuelo y Fucha. (70 \% del Volafl).

CeflST: concentración de los vertimientos domésticos en los ríos Tunjuelo y Fucha de los parámetros que se van a evaluar (DBO5, SST), reportados por el Observatorio Ambiental de Bogotá (OAB).

VoleflPTAR: volumen de vertimientos en la cuenca del río Salitre, después del tratamiento en la planta de tratamiento de aguas residuales (PTAR) el Salitre (30\% del Volafl).

CeflPTAR: concentración de los vertimientos que salen de la PTAR el Salitre después de su tratamiento en los parámetros DBO5, SST, reportados por la EAB.

Volafl: volumen de agua vertida por los habitantes de la ciudad $(85 \%$ del agua potable consumida en uso doméstico).

Cafl: concentración de los parámetros DBO5 y SST del agua potable consumida por los habitantes de la ciudad.

Cmax: los estándares calidad máximos permitidos por la autoridad ambiental. En este caso parámetros objetivo de calidad para el río Bogotá[13].

Cnat: concentración natural del río Bogotá en los parámetros DBO5 y SST.

El resultado de la ecuación se expresa en volumen/tiempo.

\subsection{Sector industrial}

Según el CTA, CSI-LAC, Cosude e Ideam, la huella hídrica del sector industrial [14] cuantifica el volumen de agua que ingresa a un proceso industrial y que finalmente queda contenido en el producto junto con los vertimientos resultantes de su fabricación por eso el sector evalúa los aportes de aguas azul y gris en su huella.

\subsubsection{Huella hídrica azul del sector industrial}

La huella hídrica azul fue cuantificada empleando el volumen de agua destinada a las actividades productivas de la ciudad. Este volumen es diferenciado en función del tipo de fuente de abastecimiento del cual provenga. En el caso de las fuentes superficiales se tuvo en cuenta el volumen facturado por la EAB y los acueductos privados, además del agua en bloque distribuida durante el periodo evaluado y el consumo en distritos de riego, mientras que en cuanto a las fuentes subterráneas se contabilizó el agua procedente de las concesiones de pozos otorgadas por la SDA y por la CAR. La información consolidada se presenta en la siguiente tabla.

Tabla 1. Volumen de agua consumida en Bogotá, 2014.

\begin{tabular}{|l|l|r|}
\hline $\begin{array}{l}\text { Tipo de fuente } \\
\text { hídrica de } \\
\text { abastecimiento }\end{array}$ & Descripción & \multicolumn{1}{|c|}{$\begin{array}{l}\text { Consumo } \\
\text { (m3/año) }\end{array}$} \\
\hline Superficial & $\begin{array}{l}\text { Acueducto - } \\
\text { EAB }\end{array}$ & $13^{\prime}$ '492.699,80 \\
\cline { 2 - 3 } & $\begin{array}{l}\text { Acueductos, } \\
\text { agua lluvia }\end{array}$ & $9^{\prime} 623.306,42$ \\
\cline { 2 - 3 } & Agua en bloque & $31.170,00$ \\
\cline { 2 - 3 } & $\begin{array}{l}\text { Distritos de } \\
\text { riego }\end{array}$ & $87.047,00$ \\
\hline Subterránea & Pozos - SDA & $4^{\prime} 220.367,20$ \\
\cline { 2 - 3 } & Pozos - CAR & $250.080,48$ \\
\hline
\end{tabular}

La información mencionada se aplicó a la resolución de la siguiente ecuación,

【WF】_Azul=afluente-efluente

Donde:

Afluente: volumen de agua destinada a las actividades industriales. Corresponde al suministro proveniente tanto de fuentes superficiales como subterráneas presentadas en la tabla 1.

Efluente: volumen de agua descargada posteriormente a su utilización. Debido a la hidrografía de la ciudad donde las cuencas abastecedoras y de recepción de vertimientos 
no son las mismas, es posible asumir que el efluente de la cuenca es cero.

\subsubsection{Huella hídrica gris del sector industrial}

La huella hídrica gris perteneciente al sector industrial se calculó mediante la ecuación 7 que corresponde al cálculo estandarizado por Hoekstra [4], en el se evalúa la capacidad de asimilación de la cuenca frente a la carga contaminante aportada.

$$
\text { [WF }]_{-}(\mathrm{Gris})=\mathrm{L} /\left(\mathrm{C} \_ \text {max }-\mathrm{C} \_ \text {nat }\right)
$$

Donde:

L: carga contaminante aportada por el sector industrial.

C_max: concentración máxima permitida para la cuenca del río Bogotá como meta para el año 2020, con base en los objetivos de calidad de agua expedidos por la CAR [13].

C_nat: parámetros fisicoquímicos del recurso hídrico, en caso de que no existan perturbaciones humanas en la cuenca. En caso de que no se cuente con los parámetros naturales, que se cree que son bajos, es posible asumir este valor como cero.

Se emplearon los datos asociados a la carga contaminante reportados por el sector industrial a la SDA mediante el registro único ambiental (RUA), donde se registraron las características de las aguas residuales de tipo industrial de 1.122 establecimientos de los 8.991 que generan vertimientos en la ciudad de Bogotá [15]. De este sectorse seleccionó una muestra de 96 empresas que contaban con datos de los parámetros de DBO5 y SST, con el fin de estandarizar los parámetros utilizados en la estimación de la huella. Posteriormente la muestra se extrapoló para el $100 \%$ de las industrias generadoras de vertimientos en el distrito capital.

Una vez realizada la estimación con cada uno de los parámetros seleccionados se tomó el resultado de mayor magnitud como huella hídrica gris para el sector.

La huella hídrica total del sector consiste en la sumatoria de las huellas azul y gris del mismo sector, como se muestra en la siguiente ecuación:

$$
\left.[\mathrm{WF}) \_\mathrm{Ind}=\llbracket[\mathrm{WF}) \_\mathrm{Azul}+\mathrm{WF}\right){ }_{-}(\mathrm{Gris})
$$

\subsection{Sector alimentos}

El consumo del recurso hídrico del sector de alimentos se cuantifica mediante la huella hídrica indirecta de los productos consumidos en el área de estudio, según la ecuación 9. Para esto, se tienen en cuenta diversos grupos de alimentos, junto a su equivalencia hídrica, que fue determinada por la Organización de las Naciones Unidas para la Alimentación y la Agricultura (FAO).

$$
[\mathrm{WF}) \_ \text {alimentos }=\sum \_\mathrm{p}\left[\left(\mathrm{Cp}^{*}[(\mathrm{EqH})] \_\mathrm{prod}\right)\right]
$$

Donde:

Cp: cantidad de productos consumidos.

$[\mathrm{EqH}) \_$prod: huella hídrica equivalente del producto.

La huella hídrica del sector de alimentos se obtuvo a partir de la información de consumo de productos agrícolas, de origen animal y de algunos productos procesados en la ciudad. Los datos reportados por el DANE [16] corresponden a la central de abastos Corabastos, la cual suple el $30 \%$ de la demanda alimenticia de la ciudad de Bogotá, esto con base en las estimaciones del DANE [16].

La muestra de alimentos empleada para realizar el cálculo de la huella hídrica fue seleccionada a partir del seguimiento realizado por el Dane [16] a 144 productos de la central de abastos Corabastos los cuales equivalen a 1'759.035 toneladas. Esta muestra fue seleccionada a partir de su existencia en la base de datos de equivalentes hídricos de la Water Footprint Network (WFN) [17], por lo cual en el cálculo fueron incluidos 126 tipos de productos que corresponden 1'749. 019 toneladas en el año 2014, que a su vez representan el 99,43\% del total de datos aportados por el Dane (tabla 2).

La equivalencia hídrica establecida por FAO en los estudios realizados por Hoekstra y Mekonnen [17] [18], mediante la metodología desarrollada por ellos, se ejecutó empleando el modelo Cropwat 8.0 para calcular las necesidades hídricas de los cultivos y las necesidades de riego con base en datos del suelo, del clima y del tipo de cosecha [17]. La huella hídrica gris de la producción agrícola y sus derivados se basa en un indicador del volumen de la contaminación de agua dulce, que es estimada con base en la cuantificación del volumen de agua necesario para asimilar los nutrientes que alcanzan fuentes de hídricas superficiales o subterráneas [17]. 
Tabla 2. Distribución de alimentos en Corabastos, 2014.

\begin{tabular}{|l|r|r|r|r|}
\hline Tipo de producto & $\begin{array}{l}\text { Cantidad } \\
\text { total (Ton) }\end{array}$ & \multicolumn{1}{|l|}{ Participación \% } & \multicolumn{1}{l|}{$\begin{array}{l}\text { Cantidad } \\
\text { muestra (Ton) }\end{array}$} & $\begin{array}{c}\text { Participación } \\
\text { muestra \% }\end{array}$ \\
\hline Verduras y hortalizas & $652.586,00$ & 37,10 & $652.586,00$ & 37,10 \\
\hline Tubérculos, raíces y plátanos & $512.129,00$ & 29,11 & $512.129,00$ & 29,11 \\
\hline Frutas & $452.568,00$ & 25,73 & $452.568,00$ & 25,73 \\
\hline Granos y cereales & $76.493,00$ & 4,35 & $76.493,00$ & 4,35 \\
\hline Procesados & $60.273,00$ & 3,43 & $51.984,00$ & 2,96 \\
\hline Lácteos y huevos & $1.972,00$ & 0,11 & $1.972,00$ & 0,11 \\
\hline Carnes & $1.431,00$ & 0,08 & $1.287,00$ & 0,07 \\
\hline Pescados & $1.583,00$ & 0,09 & & - \\
\hline
\end{tabular}

Fuente: Departamento Administrativo Nacional de Estadística (Dane).

\subsection{Disposición de residuos sólidos}

La descomposición de residuos sólidos en el relleno sanitario Doña Juana produce lixiviados diariamente. Este volumen es tratado en la planta de tratamiento de lixiviados y genera una huella hídrica gris, que se calculó de la siguiente manera:

$$
\text { 【WPPTL】_(Gris })=\mathrm{L} /\left(\mathrm{C}_{\max }-\mathrm{C}_{\text {nat }}\right)
$$

Donde:

L: concentraciones mensuales de grasa y aceites, DBO5 y fenoles de los lixiviados tratados, el caudal y el volumen tratado mensualmente, reportado por la Unidad Administrativa Especial de Servicios Públicos (UAESP) [19].

$\mathrm{C}_{\max }$ : concentración máxima permitida por la autoridad ambiental para los parámetros de calidad evaluados [13]

$\mathrm{C}_{\text {nat: }}$ concentración natural del río Tunjuelo.

\subsection{Análisis de sostenibilidad de la huella hídrica}

\subsubsection{Análisis de la sostenibilidad de la huella hídrica azul}

La huella hídrica de la cuidad se evalúa con base en la disponibilidad de agua azul mensual o agua disponible real utilizada para uso y consumo dentro la ciudad, mediante la comparación de ambas. Lo anterior se realiza por medio de la siguiente ecuación:

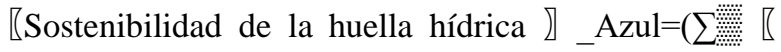
huella hídrica azul』 )/(Disponibilidad natural)
Según los resultados obtenidos es posible realizar la evaluación del impacto ambiental de la huella hídrica sobre la ciudad teniendo en cuenta los siguientes rangos:

Tabla 3. Rangos de evaluación del impacto de la huella hídrica azul.

\begin{tabular}{|l|l|}
\hline Rangos & Interpretación \\
\hline Mayor que 1 & Bajo \\
\hline Mayor que 1.5 & Moderado \\
\hline Mayor que 2 & Alto \\
\hline Mayor que 4 & Muy alto \\
\hline
\end{tabular}

Fuente: [13].

** Si la división es menor que 1, entonces se puede concluir que el impacto ambiental en cuanto al consumo de agua no existe o no es significativo.

\subsubsection{Análisis de la sostenibilidad de la huella hídrica gris}

La sostenibilidad de la huella hídrica gris, se cuantifica con el volumen de agua natural y la huella hídrica gris total de la ciudad. Este valor se denomina índice de contaminación hídrica.

Índice de contaminación hídrica=(huella gris total $) /($ disponibilidad real de agua $) * 100$

Para la evaluación del impacto de las huellas hídricas azul y gris se tomó como referencia el suministro confiable continuo (SCC) de agua que corresponde a $17,04 \mathrm{~m}^{3} / \mathrm{s}$ reportados por la EAB [12]. 


\section{Resultados y discusión}

\subsection{Sector doméstico}

3.1.1. Huella hídrica azul del sector doméstico La huella hídrica azul del sector doméstico estimada para las etapas de potabilización, distribución y consumo de agua corresponde a 488,81 millones de $\mathrm{m}^{3}$ para el año 2014, la cual está distribuida entre huella interna y externa de la siguiente manera.

Tabla 4. Huella hídrica azul sector doméstico Bogotá D.C. 2014.

\begin{tabular}{|c|c|c|c|c|c|}
\hline $\begin{array}{l}\text { Tipo de fuente hídrica de } \\
\text { abastecimiento }\end{array}$ & Descripción & $\begin{array}{c}\text { Medio de } \\
\text { abastecimiento }\end{array}$ & $\begin{array}{c}\text { Huella externa } \\
\left(\mathrm{m}^{3} / \mathbf{a} \tilde{n} o\right)\end{array}$ & $\begin{array}{c}\text { Huella interna } \\
\left(\mathbf{m}^{3} / \mathbf{a n ̃ o}\right)\end{array}$ & $\begin{array}{c}\text { Fuente de } \\
\text { información }\end{array}$ \\
\hline \multirow{4}{*}{ Superficial } & Volumen facturado & EAB & $262 ’ 764.404,00$ & & \multirow{4}{*}{ EAB } \\
\hline & Volumen no Facturado & EAB & $160 ’ 220.000,00$ & & \\
\hline & Agua en bloque & EAB & $29^{\prime} 280.000,00$ & & \\
\hline & $\begin{array}{l}\text { Consumo para } \\
\text { potabilización }\end{array}$ & EAB & $15^{\prime} 147.507,00$ & & \\
\hline \multirow{2}{*}{ Subterránea } & Pozos & Concesión SDA & & $400.809,00$ & SDA \\
\hline & Pozos & Concesión CAR & & $20 ’ 999.759,33$ & CAR \\
\hline \multicolumn{3}{|l|}{ Subtotal } & $467^{\prime} 411.911,00$ & $21^{\prime} 400.568,33$ & \\
\hline \multicolumn{3}{|l|}{ Total } & \multicolumn{2}{|r|}{$488^{\prime} 812.479,33$} & \\
\hline
\end{tabular}

Fuente: autores.

El $55 \%$ de la huella hídrica azul del sector doméstico corresponde a 262.76 millones de $\mathrm{m}^{3}$ de agua al año, los cuales son transportados a los usuarios a través del sistema de acueducto que factura por el servicio, sin embargo, en este proceso se generan pérdidas tanto técnicas como comerciales, que pueden ser por fallas en las líneas de conducción y distribución, en los tanques de almacenamiento o producto de acciones ilegales. Estas pérdidas contabilizadas como volumen no facturado alcanzan los 160.220 millones de $\mathrm{m}^{3}$ al año (tabla 4), y representan cerca del $33 \%$ de la huella hídrica azul del sector (figura 1).

Tabla 5. Volumen de vertimientos y concentración Bogotá D.C., 2014.

\begin{tabular}{|c|c|c|c|c|c|}
\hline \multirow[b]{2}{*}{ Huella hídrica (HH) } & \multirow[b]{2}{*}{ Volumen (m3) } & \multirow{2}{*}{$\begin{array}{l}\text { Carga contaminante } \\
\text { SST (Ton/año) }\end{array}$} & \multirow{2}{*}{$\begin{array}{c}\text { Carga } \\
\text { contaminante DBO } \\
\text { (Ton/año) } \\
\end{array}$} & \multicolumn{2}{|c|}{ Huella hídrica gris año } \\
\hline & & & & $\operatorname{SST}\left(\mathbf{m}^{3}\right)$ & $\operatorname{DBO5}\left(\mathrm{m}^{3}\right)$ \\
\hline $\mathrm{HH}$ sin tratamiento & $293 ’ 420.927,65$ & $66.152,81$ & $62.315,90$ & $1.653^{\prime} 820.497,87$ & $1.246 ’ 318.187,13$ \\
\hline HH tratamiento PTAR Salitre & $124 ’ 341.670,00$ & $23.357,93$ & $14.208,07$ & 583'948.190,53 & $284^{\prime} 161.539,86$ \\
\hline Total & $417^{\prime} 762.597,65$ & & & $2.237^{\prime} 768.688,39$ & $1.530 ’ 479.726,99$ \\
\hline
\end{tabular}

Fuente: autores.

\subsubsection{Huella hídrica gris del sector doméstico}

La huella hídrica gris del sector doméstico corresponde al resultado de la máxima entre las dos huellas evaluadas (tabla 5), siendo este valor el de SST.

La Huella Hídrica Gris del sector se valoró en 2237.76 millones de $\mathrm{m} 3$, equivalente a la cantidad de SST presentes en los ríos Tunjuelo, Salitre, Fucha y Torca. Por los vertimientos de uso doméstico que son transportados al río Bogotá cada usuario del servicio de alcantarillado debe pagar la tasa retributiva equivalente según su estrato.

\subsubsection{Huella hídrica total del sector doméstico}

La huella hídrica total del sector doméstico de la ciudad en el año 2014 fue estimada en 2.726,58 millones de $\mathrm{m}^{3}$, de los cuales el $82 \%$ pertenece a huella gris y $18 \%$ a huella azul (figura 2). Esta diferencia se debe a la carga contaminante presente en los vertimientos, pues se necesitaría mayor cantidad de agua para devolverlos a los parámetros de calidad iniciales.

Se evidenció que la carga contaminante aportada por los vertimientos los ríos Tunjuelo y Fucha que serán tributados al río Bogotá son de mayor magnitud que los del río Salitre. Esto está directamente relacionado con la presencia de la PTAR, que logra remover una parte de la carga. Esto altera significativamente el volumen de la huella de la ciudad. 
Figura 1. Porcentaje de participación en la huella hidrica azul del sector doméstico de Bogotá, 2014.

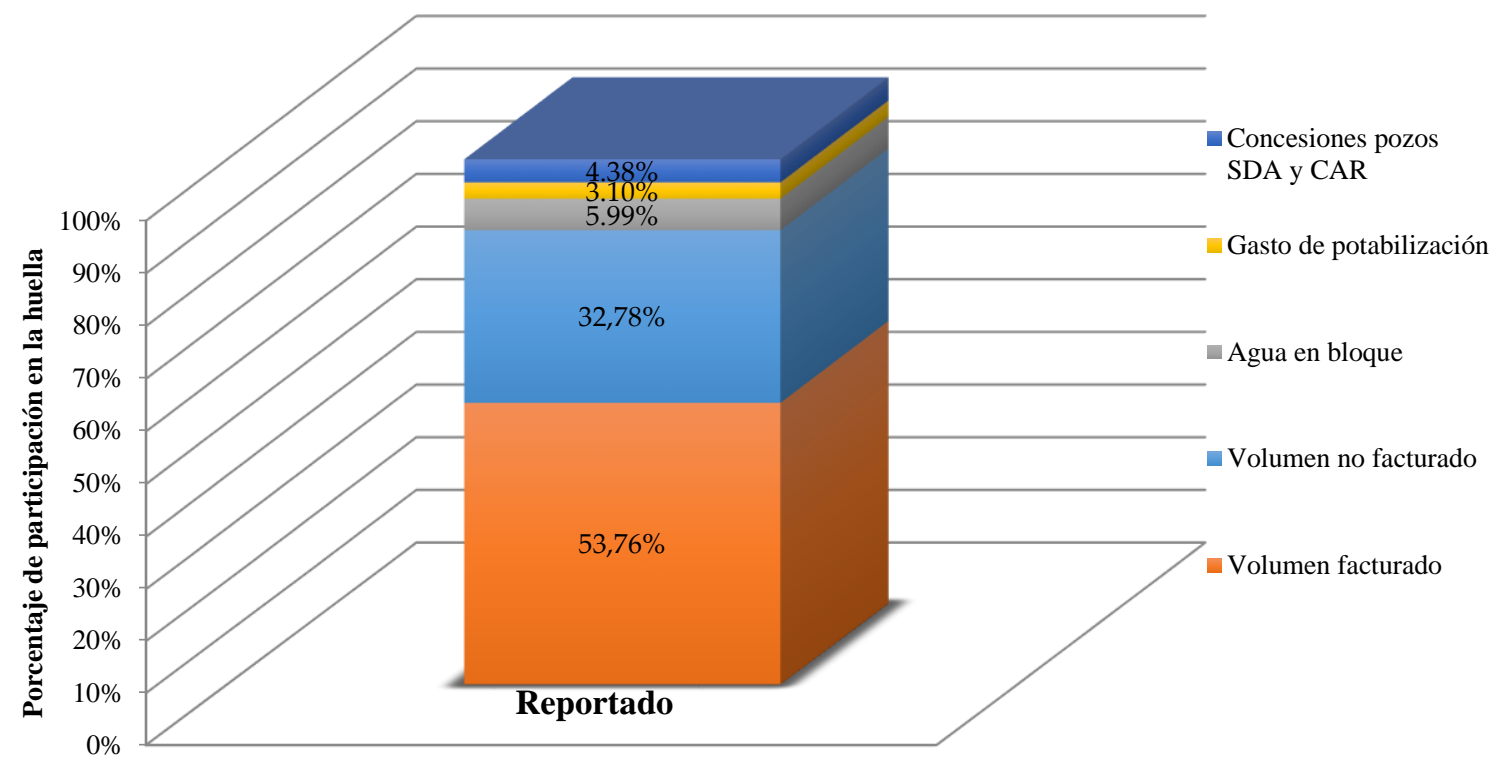

Fuente: autores.

Figura 2. Porcentaje de participación en la huella hídrica total del sector doméstico de Bogotá, 2014.

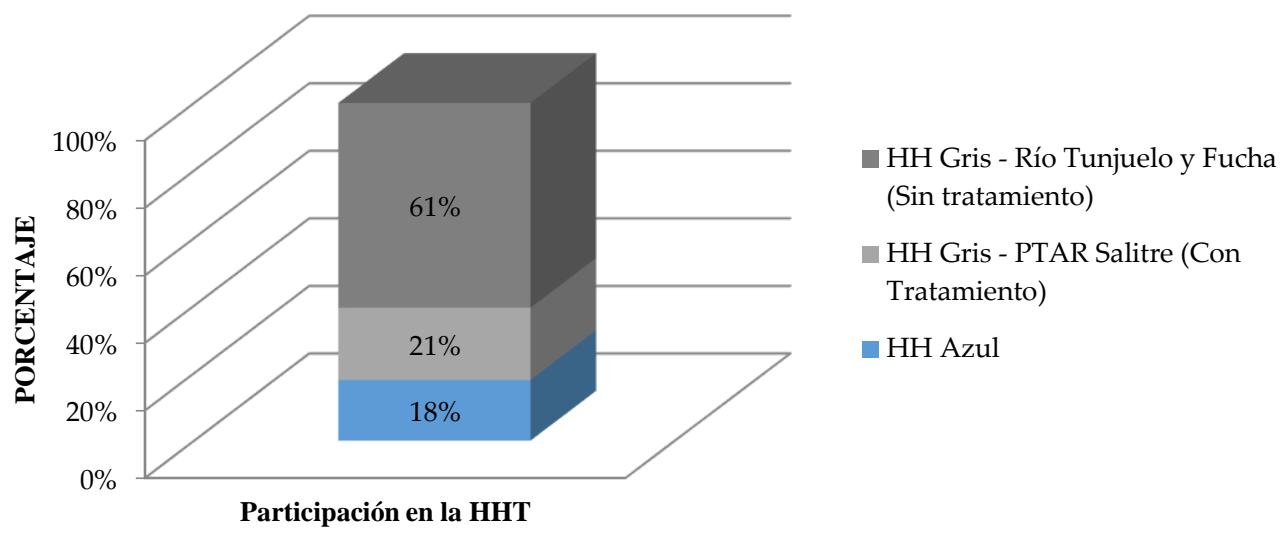

Fuente: autores.

\subsection{Sector Industrial}

La huella hídrica azul correspondiente al sector industrial es de 27,70 (figura 3) millones de $\mathrm{m}^{3} /$ año de los cuales el $49,13 \%$ corresponde a agua virtual y el restante $50,87 \%$ se debe a volúmenes de agua captada de las fuentes internas de la ciudad. Estas fuentes también son utilizadas y pertenecen a municipios adyacentes como consecuencia de las formaciones hidrogeológicas de la región.

De otra parte, el aporte de agua gris del sector industrial es de 873,73 millones de $\mathrm{m}^{3}$ /año del cual el 12,48\% corresponde al sector industrial controlado por la SDA y el $87,52 \%$ es aportado por industrias que no cuentan con registro de vertimientos, como se aprecia en tabla 6 . 
Figura 3. Huella azul interna y externa del sector industrial de Bogotá y controlado por la SDA - 2014.

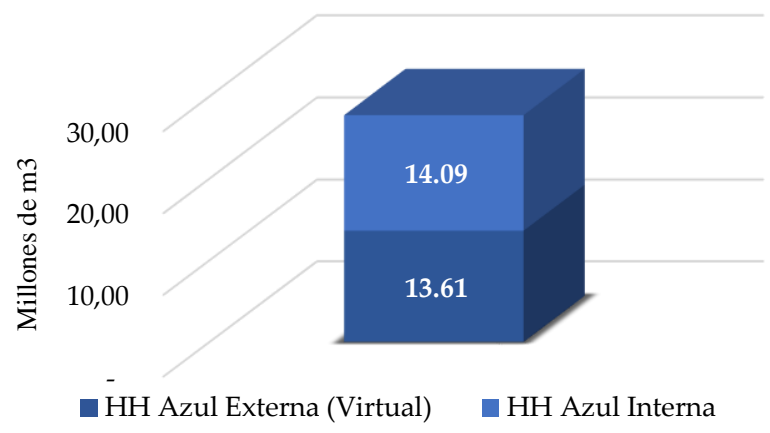

Fuente: autores.

Tabla 6. Huella hídrica gris sector industrial de Bogotá.

\begin{tabular}{|c|c|c|c|c|c|c|}
\hline \multicolumn{4}{|c|}{ Industrias } & & & \\
\hline Tipo & $\mathrm{n}^{\circ}$ & Muestra & $\%$ & DBO (5 días) & SST & $\begin{array}{l}\text { Huella hídrica total } \\
\left(\mathrm{m}^{3} / \mathrm{a} \tilde{\mathrm{a}} \mathrm{o}\right)\end{array}$ \\
\hline Con registro & 1.122 & & 107 & 2'320 185 & 2'080 01706 & \multirow{3}{*}{$873^{\prime} 736.504,35$} \\
\hline Sin registro & 7.869 & 96 & 1,07 & 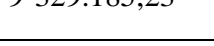 & סוז, & \\
\hline Total & \multicolumn{2}{|l|}{8.991} & 100 & $873 ’ 736.504,35$ & $373 ’ 680.754,05$ & \\
\hline
\end{tabular}

Fuente: autores.

Como fue mencionado anteriormente el sector industrial consumió 27,70 millones de $\mathrm{m}^{3} /$ año, que corresponden a agua azul, y que después se transformaron en vertimientos que requieren volúmenes de agua mayores para lograr la dilución de la carga contaminante y así regresar el agua empleada a los parámetros de calidad fijados por la legislación[13]. Se debe tener en cuenta que el parámetro que representa la huella hídrica es la DBO (5). A partir de lo anterior se realizó el cálculo de la huella total del sector, que equivale a 901,44 millones de $\mathrm{m}^{3} / \mathrm{a} \tilde{\text { no. }}$

\subsection{Sector alimentos}

La huella hídrica de la muestra seleccionada para el sector de alimentos es de 1.358,76 millones de $\mathrm{m}^{3} /$ ton/año que corresponden al $99,43 \%$ de los alimentos suministrados por la central de abastos. Por tanto, la huella del total de los alimentos provenientes de Corabastos en Bogotá es de 1.366 millones de $\mathrm{m}^{3} /$ ton/año. Con base en lo anterior y el porcentaje de abastecimiento reportado por el Dane [16] de la central de abastos que se encuentra alrededor del $30 \%$ de la demanda de los habitantes de la ciudad, se realizó una extrapolación con el fin de determinar la huella hídrica total del sector para el año 2014, que equivale 4.555 millones de $\mathrm{m}^{3} /$ ton/año.

La huella hídrica del sector de alimentos de Bogotá en el año 2014 se compone en un $38.9 \%$ por aportes del grupo de verduras y hortalizas seguido por el grupo de las frutas con un $25.3 \%$ ya que la cantidad suministrada de productos pertenecientes a estos grupos de alimentos por parte de la central de abastos es significativamente mayor a la cantidad productos de origen animal como carnes, huevos y lácteos.

La huella hídrica total para el sector de alimentos de la ciudad de Bogotá en el año 2014 está constituida en mayor proporción por agua verde, la cual aporta el $82 \%$ del volumen empleado en el sector, donde la mayor parte se debe a la incorporación de agua natural a los cultivos o al consumo de forma indirecta debido a la demanda nutricional de los animales, La proporción de agua azul empleada en el sector sea esto se encuentra ligada la proporción de agua azul empleada en el sector, la cual corresponde al $7 \%$ de total de la huella. Esta, la relación se debe a la utilización de riego como actividad complementaria en la producción de los alimentos consumidos por los habitantes de la ciudad y en los 
usados lo usado como insumos para la producción de alimentos de origen animal, además del agua empleada en el ciclo productivo de los alimentos procesados. Finalmente, la huella gris aporta un $11 \%$ a la huella del sector, y se encuentra repartida en contribuciones del

Figura 4. Aporte a la huella hídrica por grupo de alimentos, 2014.

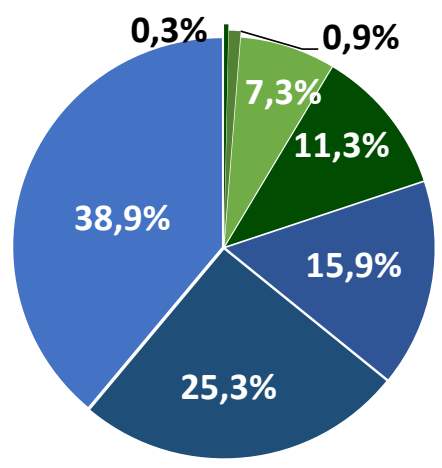

sector agrícola por parte de los fertilizantes, plaguicidas y defoliantes empleados y del sector pecuario, que constituyen los desechos generados por el ganado productor (figura 5).

Fuente: autores.

Figura 5. Huella hídrica sector de alimentos, 2014.

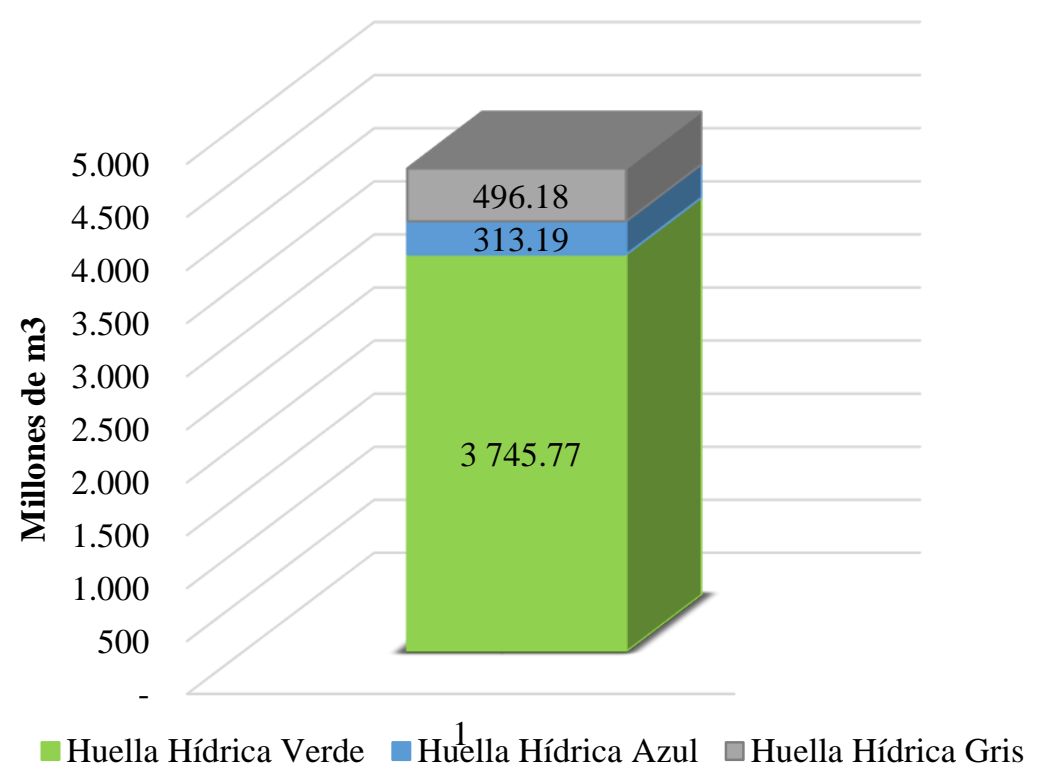

Fuente: autores.

Teniendo en cuenta que la huella correspondiente a este sector se basa en un enfoque de consumo es posible afirmar que la huella hídrica con relación a los habitantes de la ciudad de Bogotá es aproximadamente de 1.642,1 /habitante/día, teniendo en cuenta la encuesta multipropósito realizada por la Secretaría Distrital de

\author{
- Lácteos y huevos \\ carnes \\ - Procesados \\ - Tubérculos, raíces y plátanos \\ - Granos y cereales \\ - Frutas \\ - Verduras y hortalizas
}


lixiviados. La planta de tratamiento de lixiviados Doña Juana trató caudales promedio mensuales entre 9,48 y 23,01 1/s. [19]. La huella hídrica de mayor relevancia fue la presentada por grasas y aceites, se estimó en 1.306,42 millones de $\mathrm{m}^{3}$ al año.

\subsection{Análisis de sostenibilidad de la huella hídrica azul y gris}

Según lo propuesto por Hoekstra se puede concluir que el impacto ambiental en cuanto al consumo de agua azul es moderado. En cuanto al impacto de la huella hídrica gris se puede considerar que se necesita 9 veces la cantidad de agua de abastecimiento para asimilar la carga de contaminantes dados las concentraciones naturales y estándares ambientales de calidad de agua.

Tabla 8. Análisis de sostenibilidad de la huella hídrica

\begin{tabular}{|l|l|}
\hline Sostenibilidad de la huella azul & 1,54 \\
\hline Índice de contaminación hídrica & 9,14 \\
\hline
\end{tabular}

Fuente: autores.

Tabla 7. Huella hídrica gris sector de disposición de residuos solidos.

\begin{tabular}{|c|c|c|c|c|c|c|c|}
\hline \multirow[b]{2}{*}{ Mes } & \multirow{2}{*}{$\begin{array}{c}\mathbf{Q} \\
\text { tratado } \\
\mathbf{m}^{3} / \mathrm{seg}\end{array}$} & \multicolumn{3}{|c|}{ Concentración del vertimiento } & \multicolumn{3}{|c|}{ Huella hídrica gris } \\
\hline & & $\begin{array}{l}\text { DBO5 } \\
\left(\mathrm{g} / \mathrm{m}^{3}\right)\end{array}$ & $\begin{array}{c}\text { Grasas y } \\
\text { Aceites } \\
\left(\mathbf{g} / \mathbf{m}^{3}\right) \\
\end{array}$ & $\begin{array}{c}\text { Fenoles } \\
\left(\mathrm{g} / \mathbf{m}^{3}\right)\end{array}$ & DBO $\left(\mathbf{m}^{3} / \mathrm{seg}\right)$ & $\begin{array}{c}\text { Grasa y aceites } \\
\left(\mathbf{m}^{3} / \mathrm{seg}\right)\end{array}$ & $\begin{array}{l}\text { Fenoles } \\
\left(\mathrm{m}^{3} / \mathrm{seg}\right)\end{array}$ \\
\hline Ene. & 0,38 & 44,45 & 2,10 & 0,03 & 0,17 & 0,40 & 0,06 \\
\hline Feb. & 0,36 & 37,98 & 2,13 & 0,02 & 0,14 & 0,38 & 0,04 \\
\hline Mar. & 0,40 & 26,45 & 4,58 & 0,06 & 0,11 & 0,92 & 0,12 \\
\hline Abr. & 0,39 & 25,00 & 6,38 & 0,06 & 0,10 & 1,25 & 0,12 \\
\hline May. & 0,24 & 43,83 & 5,23 & 0,04 & 0,11 & 0,63 & 0,05 \\
\hline Jun. & 0,39 & 469,50 & 11,78 & 0,17 & 1,82 & 2,29 & 0,32 \\
\hline Jul. & 0,67 & $1.313,00$ & 42,82 & 0,27 & 8,80 & 14,35 & 0,89 \\
\hline Ago. & 0,61 & 949,00 & 25,98 & 0,88 & 5,77 & 7,89 & 2,66 \\
\hline Sep. & 0,48 & 677,00 & 14,50 & 0,35 & 3,26 & 3,49 & 0,84 \\
\hline Oct. & 0.48 & $1.023,00$ & 17,50 & 0,68 & 4,94 & 4,22 & 1,65 \\
\hline Nov. & 0.35 & $1.143,00$ & 6,20 & 0,15 & 3,95 & 1,07 & 0,26 \\
\hline Dic. & 0.36 & $1.211,00$ & 24,90 & 0,07 & 4,40 & 4,53 & 0,13 \\
\hline \multicolumn{5}{|c|}{ Total HH (m³/seg) } & 33,56 & 41,43 & 7,14 \\
\hline \multicolumn{5}{|c|}{ Total HH (mªño) } & $1.058 ' 295.829,80$ & $1.306^{\prime} 422.813,16$ & $225 ’ 026.927,95$ \\
\hline
\end{tabular}

Fuente: UAESP, 2015 Cálculos: autores.

\subsection{Huella hídrica total}

La huella hídrica de la ciudad de Bogotá de los sectores doméstico, industrial, alimentario y de residuos sólidos en el año 2014 correspondió a 9.489'587.694,93 $\mathrm{m}^{3}$. De ellos el sector que más aporta es el agrícola, seguido por el doméstico, el de residuos sólidos y el industrial.

La huella hídrica para la ciudad de Bogotá está compuesta en mayor proporción por aportes de agua gris con el $51,78 \%$ de los mismos, estos son generados principalmente por el sector doméstico, seguidos por el volumen de agua requerido en la disposición de residuos sólidos y el sector industrial, mientras que el volumen de agua verde 39,47\% de la huella hídrica, se debe en gran parte a las actividades relacionadas con el sector alimentario, que a su vez es el que más contribuye a la huella total de la ciudad. El componente con menor volumen dentro de la huella hídrica corresponde al agua captada de fuentes hídricas superficiales, destinada al cubrimiento de la demanda de los diferentes sectores de la ciudad, que aporta el 8,75\% restante (figura 6). 
Tabla 9. Huella hídrica multisectorial de Bogotá, año 2014.

\begin{tabular}{|c|r|r|r|r|r|r|r|r|}
\hline Sector & $\begin{array}{c}\text { Huella } \\
\text { azul } \mathbf{~ m 3}\end{array}$ & $\begin{array}{c}\text { \% del } \\
\text { total }\end{array}$ & $\begin{array}{c}\text { Huella gris } \\
\mathbf{m 3}\end{array}$ & $\begin{array}{c}\text { \% del } \\
\text { total }\end{array}$ & $\begin{array}{c}\text { Huella } \\
\text { verde } \mathbf{~ m 3}\end{array}$ & \% del total & $\begin{array}{c}\text { Huella total } \\
\text { millones de m3 }\end{array}$ & \% del total \\
\hline Agrícola & 313,19 & $3,30 \%$ & 496,18 & $5,23 \%$ & $3.745,77$ & $39,47 \%$ & $4.555,14$ & $48,00 \%$ \\
\hline Doméstico & 488,81 & $5,15 \%$ & $2.237,77$ & $23,58 \%$ & 0,00 & $0,00 \%$ & $2.726,58$ & $28,73 \%$ \\
\hline Industrial & 27,70 & $0,29 \%$ & 873,74 & $9,21 \%$ & 0,00 & $0,00 \%$ & 901,44 & $9,50 \%$ \\
\hline RSDJ & 0,00 & $0,00 \%$ & $1.306,42$ & $13,77 \%$ & 0,00 & $0,00 \%$ & $1.306,42$ & $13,77 \%$ \\
\hline Todos los sectores & 829,71 & $8,74 \%$ & $4.914,10$ & $51,78 \%$ & $3.745,77$ & $39,47 \%$ & $9.489,59$ & $100,00 \%$ \\
\hline
\end{tabular}

Fuente: autores.

Los resultados muestran la gran importancia que reviste el tratamiento de las aguas residuales y de los vertimientos de lixiviados para reducir la huella hídrica gris y por esta vía la huella hídrica total de la ciudad.

Figura 6. Huella hídrica total para la ciudad de Bogotá D.C.

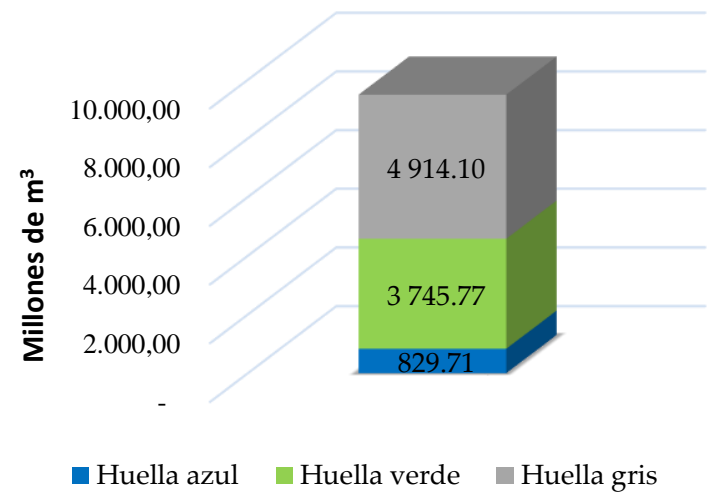

Fuente: autores.

La huella per cápita en la ciudad es de 1.217,48 m³/año según la encuesta multipropósito realizada por la Secretaría Distrital de Planeación [20] para el año 2014.

Para realizar una comparación adecuada con los resultados obtenidos en otras áreas geográficamente definidas es necesario incluir la totalidad de los sectores involucrados, sin embargo, cabe mencionar que la huella de países latinoamericanos como Brasil y México se encuentra alrededor de $1.381,32$ y $1.357,31 \mathrm{~m}^{3} /$ año por habitante para el año 2014. Según Conagua[10], con base en el comportamiento de Bogotá como ciudad principal, Colombia se ubica en una posición cercana a las de Brasil y México.

\section{Conclusiones}

La aproximación a la estimación de la huella hídrica de Bogotá permitió examinar de manera general el manejo, la gestión pública del agua en la ciudad y el uso del recurso para desarrollar actividades económicas, productivas y de servicios en diferentes sectores de la ciudad.

Se evidenció la falta de información asociada al uso del recurso hídrico en la ciudad por parte de las entidades responsables de su administración, como los casos de la SDA, que solo tiene conocimiento de la cantidad de agua que consumen y los vertimientos que 1.122 establecimientos de los 8. 991 presentes en la ciudad, y de la $\mathrm{EAB}$, que tiene pérdidas de agua de 160,22 millones de $\mathrm{m}^{3}$ por fallas en el sistema de distribución, acciones ilegales y fraudes en los medidores, de los que se desconoce su ubicación. Así mismo, la huella hídrica per cápita de la ciudad es bastante alta comparada con ciudades de similar población, a pesar de las cuantiosas sumas de dinero que invierten la SDA y la EAB para la gestión del recurso.

De igual manera, la estimación de la cantidad del recurso hídrico consumido en la ciudad permitió comparar los patrones de uso del agua entre sectores. Esto, reveló que la mayor cantidad de recurso aprovechado está compuesto por el consumo de alimentos con un $48 \%$ del total de la huella, porcentajes que provienen en su mayoría de otras regiones del país. También se identificó que este consumo, sumado a otros productos generan residuos sólidos y a su vez lixiviados cuando son dispuestos en el relleno sanitario Doña Juana y representan el 13,77\% del total de la huella. Es decir, que sólo el consumo de productos y la generación de residuos sumados constituyen el 61, 77 \% de la Huella Hídrica.

Por tipo de huella se pudo distinguir que la huella hídrica gris hace el mayor aporte, con un 51,78 \% del total, y un índice de contaminación de 9,14. Esto revela que en todos los sectores de la ciudad el recurso se contamina diariamente, y que se necesitaría nueve veces la cantidad de agua de abastecimiento para llevarla a las condiciones 
establecidas por la legislación vigente. Igualmente, se refleja la baja responsabilidad ambiental de las industrias para el tratamiento de sus vertimientos, ya que solo algunas industrias poseen planta para este fin, sumado a la incapacidad de las plantas para tratar el caudal total de vertimientos generados en la ciudad.

Así mismo, se puede considerar que aunque en el análisis de la huella hídrica azul se encontró un impacto moderado, la ciudad de Bogotá es altamente dependiente del agua de otras regiones, puesto que, en su mayoría, los alimentos consumidos no se producen dentro de la ciudad, y el agua para consumo proviene de otra cuenca, pues las fuentes de agua pertenecientes al distrito no suplen la demanda de los diferentes sectores. Esto demuestra que la ciudad de Bogotá es vulnerable en cuanto al abastecimiento de agua e insostenible frente a ese recurso, pues se suple de fuentes externas.

Con base en lo anterior el panorama que le espera a la ciudad frente al futuro del recurso hídrico es preocupante. Si se considera la dependencia del agua frente a otras regiones, el aumento de la población y sus patrones de consumo, así como el hecho de que no se le da un tratamiento adecuado a los vertimientos generados, Bogotá se enfrentaría a la posibilidad de escasez del agua, al riesgo de desabastecimiento de alimentos para el consumo en general y se pondría en riesgo la producción industrial. A esto se sumaría la responsabilidad de la ciudad por la deuda de agua no cuantificada ni valorada con otras regiones, bien sea por la importación de agua, o por la contaminación de estas fuentes.

Por último, los resultados presentados en este artículo pretenden servir como herramienta de análisis y discusión en todos los ámbitos políticos, mostrar una visión general del uso del recurso en la ciudad e indicar los sectores en los que se genera mayor huella, para que se tengan en cuenta en la priorización de medidas de manejo y se contribuya a disminuir la huella hídrica de la ciudad. De igual manera, se reitera la falta de información y la dificultad en el acceso concerniente al manejo del agua, lo que limita considerablemente los estudios y genera incertidumbre en su elaboración. Para posteriores análisis, se recomienda que se consideren otros sectores y niveles adicionales como la huella indirecta que se genera en la fabricación de diversos productos que se consumen en Bogotá, de modo que se pueda tener un inventario de cada una de las actividades que generan algún impacto ambiental.

\section{Referencias}

[1] J. M. García Fernández y L. Cantero Corrales, «Indicadores globales para la evaluación del uso sostenible del recurso agua: Caso cubano», Volunt. Hidráulica, vol. 99, pp. 1-16, 2007.

[2] Arévalo D, «Una mirada a la agricultura de Colombia desde su Huella Hídrica», Wwf, p. 67, 2012.

[3] Waterfootprint, «Manual de Evaluacion HH», 2002.

[4] M. M. Mekonnen y A. Y. Hoekstra, «The green, blue and grey water footprint of crops and derived crop products», Hydrol. Earth Syst. Sci., vol. 15, n. ${ }^{\circ}$ 5, pp. 1577-1600, 2011.

[5] S. G. Isidro, F. J. Gutiérrez Lozano, y C. M. Torres, «Vulnerabilidad, amenaza y peligro a la contaminación de las aguas subterráneas en la región de Bucaramanga.», Rev. UIS Ing., vol. 10, n. ${ }^{\circ}$ 1, pp. 51-62, 2011.

[6] C. Blanco-Mayorga; L. Donado; D.A. Barajas, "Estudio del comportamiento de solutos reactivos en acuíferos confinados bajo diferentes condiciones de cinética química", Rev. UIS Ing., vol. 8, n. ${ }^{\circ}$ 1, pp. 77-90, 2009.

[7] J. F. Schyns y A. Y. Hoekstra, «The added value of Water Footprint Assessment for national water policy: A case study for Morocco», PLoS One, vol. 9, n. ${ }^{\circ}$ 6, pp. 125, 2014.

[8] WWF, «L'empreinte eau de La France», 2012.

[9] Conagua, «Estadisticas del agua en México», 2011.

[10]«Huella hídrica en países seleccionados.», 2014. [En línea]. Disponible en: http://201.116.60.25/sina/index_jquerymobile2.html?tema=huellaHidrica.

[11]Ministerio De Desarrollo Económico, «Dirección de agua potable y saneamiento básico." REGLAMENTO TÉCNICO DEL SECTOR DE AGUA POTABLE Y SANEAMIENTO BASICO “.» p. 2000, 2000.

[12] Empresa de Acueducto y Alcantarillado de Bogotá $E A B$. Obtenido de En respuesta a requerimiento de contraloría de Bogotá mediante oficio EAB E2015042744. 2015. 2015.

[13]C. A. R. de Cundinamarca, ACUERDO NUMERO 043 DE 2006 de la Corporación Autónoma Regional de Cundinamarca, CAR. Colombia, 2006. 
[14]«Evaluación multisectorial de la huella hídrica en Colombia», 2015.

$[15] \ll$ Entrevista en respuesta a solicitud de la Contraloría de Bogotá.», 2015.

[16]DANE, «Obtenido de Respuesta a Solicitud de la Contraloría de Bogotá. 2015». 2015.

[17] a Y. M.M. Meconnen, Hoekstra, «The green, blue and grey water footprint of farm animals and animal products», Unesco, vol. 1, n. ${ }^{\circ}$ 16, p. 80, 2010.

[18]A. Y. Hoekstra, A. K. Chapagain, M. M. Aldaya, y M. M. Mekonnen, The Water Footprint Assessment Manual. 2011.

[19]«Unidad Administrativa Especial de Servicios Públicos - UAESP, En respuesta a requerimiento de contraloría de Bogotá mediante oficio UAESP 20154010063781. 2015». p. 2015, 2015.

[20] «Secretaría Distrital de Planeación - SDP. "Encuesta multipropósito 2014” 2015.» p. 2015, 2015. 